\title{
Vorbereitung des Kaninchens auf eine Allgemeinanästhesie - Wie Sie das Narkoserisiko senken können
}

Lena Carolin Voigt

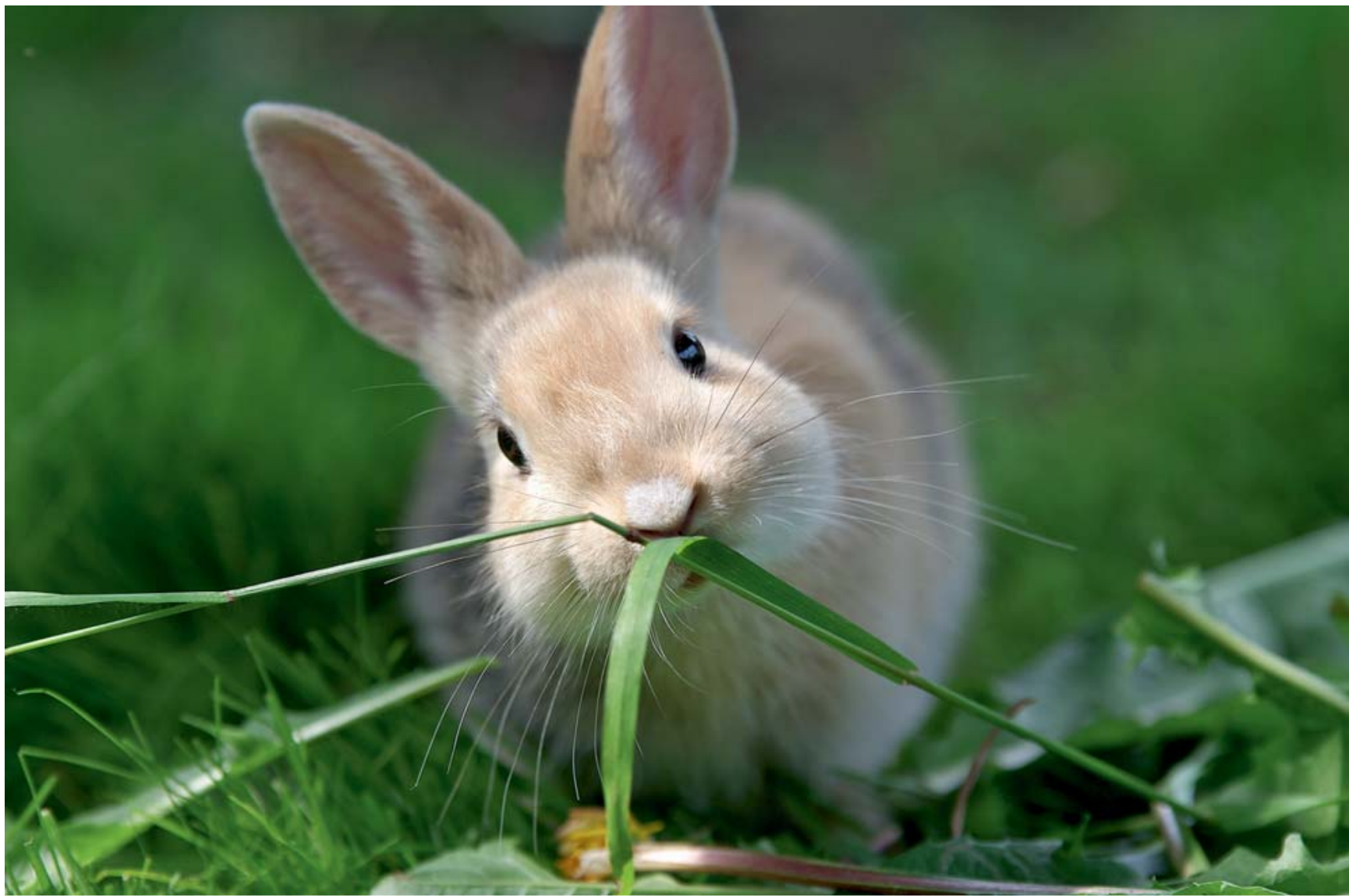

(c) cuhle-fotos - stock.adobe.com [rerif]

Die Allgemeinanästhesie des Kaninchens ist anspruchsvoller und mit einem höheren Risiko verbunden als bei Hund und Katze. Der Beitrag gibt wichtige Informationen, wie Sie das Narkoserisiko durch eine gute Vorbereitung des Patienten reduzieren können.

\section{Einleitung}

Die Zahl der vorgestellten Kaninchen in der Praxis nimmt immer weiter zu. Die Bereitschaft bzw. der Wunsch der Patientenbesitzer, therapeutisch alles erdenklich Mögliche durchzuführen, steigt immer mehr. Gleichzeitig gilt das Heimtier bei vielen Tierärzten als ein extrem hoher Risikopatient bei einer Allgemeinanästhesie und stellt viele Kollegen vor eine große Herausforderung. So bestätigen auch Studien [1], dass die anästhesiebedingte To- desrate bei Heimtieren zurzeit noch deutlich höher ist $(1,39 \%)$ als bei Hund $(0,17 \%)$ und Katze $(0,24 \%)$.

Mit den richtigen Vorbereitungen des Heimtiers auf eine Anästhesie lässt sich das Narkoserisiko bereits deutlich verringern:

- Ausschalten möglichst vieler Risikofaktoren

- individuell angepasste Protokolle

- gutes Monitoring während der Anästhesie 
Viele Faktoren führen dazu, dass die Allgemeinanästhesie beim Heimtier anspruchsvoller und risikoreicher ist als bei Hund und Katze. Heimtiere sind sehr stressempfindlich, sie reagieren bei den geringsten Veränderungen mit einer sehr starken Katecholaminausschüttung und weisen eine geringe therapeutische Breite gegenüber Anästhetika auf. Hinzu kommen anatomische Gegebenheiten, die eine Anästhesie zur Herausforderung werden lassen.

\section{Präanästhetische Untersuchung}

\section{Allgemeinuntersuchung}

Vor jedem Eingriff ist eine sorgfältige Anamnese zu erheben und eine gründliche Allgemeinuntersuchung durchzuführen. So kann der Allgemeinzustand eines jeden Patienten ermittelt und bei elektiven Eingriffen vor der Anästhesie mittels adäquater Therapie verbessert werden.

\section{Merke}

Auf das kardiovaskuläre und das respiratorische System ist dabei besonderes Augenmerk zu legen.

\section{Herz und Lunge}

Viele Kaninchen leiden an subklinischen Atemwegsinfektionen. Diese können während der Narkose, aufgrund eines vermindert funktionalen Lungengewebes, zu einer respiratorischen Insuffizienz führen. Für die Auskultation des Herzens und der Lunge ( $\bullet$ Abb. 1) muss sich genügend Zeit genommen werden. Aufgrund der sehr hohen Herz- und Atemfrequenz ist es bei den Heimtieren meist sehr schwierig Unregelmäßigkeiten zu hören.

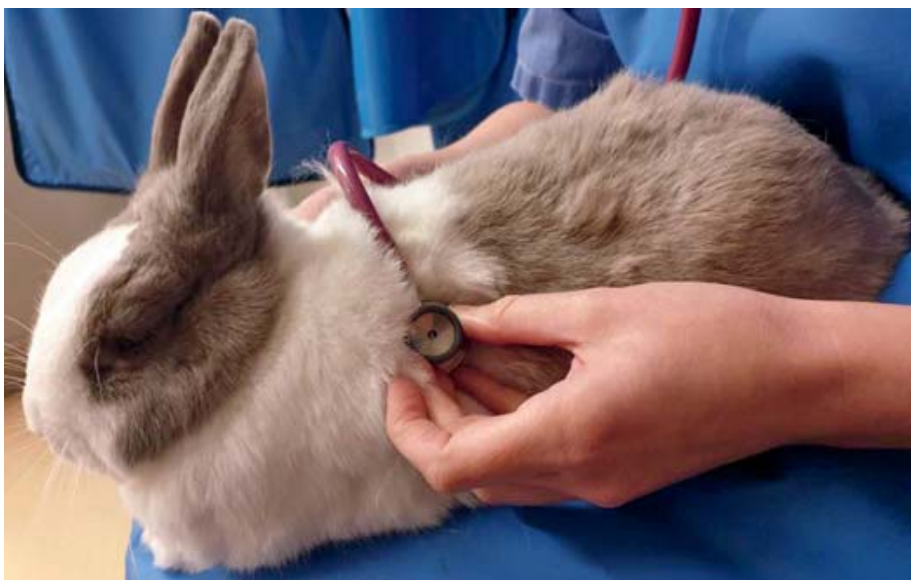

- Abb. 1 Auskultation der Lunge und des Herzens bei einem Kaninchen als Teil einer präanästhetischen Untersuchung. ( ) Lena Carolin Voigt, Klinik für Heimtiere, Reptilien, Zier- und Wildvögel, Stiftung Tierärztliche Hochschule Hannover

\section{Dehydratationszustand}

Der Dehydratationszustand sollte ebenfalls vor der Anästhesie ermittelt werden. Dies ist wichtig, um die Tiere ggf. vorher noch ausreichend mit Infusionen zu stabilisieren und um eine adäquate Flüssigkeitstherapie in der OP einstellen zu können.

\section{Körpergewicht}

Die Ermittlung des grammgenauen Gewichts ist für die exakte Dosierung der Anästhetika und der Flüssigkeitstherapie wichtig. Die Fett-Muskel-Verteilung bzw. das Verhältnis von absolutem Körpergewicht und Masse des Magen-Darm-Trakts muss dabei unbedingt mitbedacht werden. Bei kachektischen Tieren können eine eingeschränkte Leberfunktion und ein geringerer Plasmaeiweißspiegel vorliegen, sodass die Dosis deutlich reduziert werden muss. Sehr stark adipöse Tiere können aufgrund der Umverteilung der Anästhetika einen sehr langen Nachschlaf haben. Tritt eine Notfallsituation ein, ist das genaue Gewicht entscheidend für die Notfallmedikamente.

Alter

Das Alter spielt ebenfalls eine Rolle. Sehr junge Tiere haben meist ein schlechteres Temperaturregulationssystem und neigen zu Hypoglykämien, während sehr alte Tiere häufiger unter Nierenfunktionsstörungen, Herzerkrankungen oder anderen Organerkrankungen leiden.

\section{Merke}

Der Besitzer muss vor der Anästhesie über Risiken aufgeklärt werden und dies sollte dokumentiert werden.

\section{Weiterführende Untersuchungen}

Treten bei der Allgemeinuntersuchung Auffälligkeiten auf, sollten weitere Untersuchungen folgen.

\section{Auskultationsbefund}

Bei einem auffälligen Auskultationsbefund sollten auf jeden Fall zunächst Röntgenbilder der Lunge und des Herzens in 2 Ebenen ( $\triangleright$ Abb.2) durchgeführt werden, um eine Lungenerkrankung oder ein vergrößertes Herz ausschließen zu können. Bei einem unklaren Herzbefund ist es sinnvoll, ein EKG und eine Sonografie des Herzens vor der Anästhesie durchzuführen.

\section{Blutbild}

\section{Merke}

Ein großes Blutbild ( $\bullet$ Abb. 3 ) vom Patienten anzufertigen ist stets empfehlenswert.

Nur so können ein optimales Anästhesieprotokoll erstellt und mögliche Verschiebungen, zum Beispiel der Elektrolyte, noch vor der Anästhesie therapiert werden. 


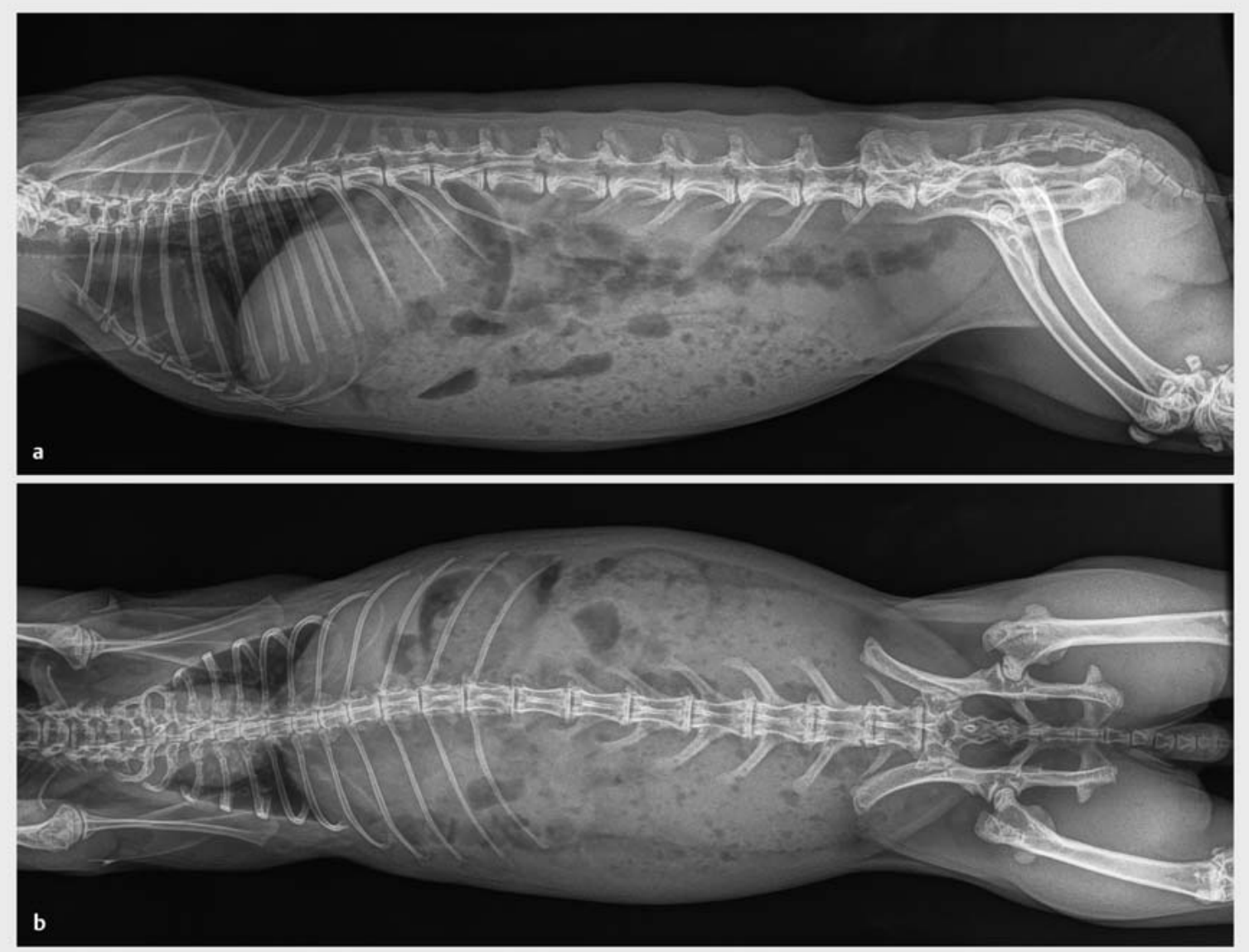

- Abb. 2 Röntgenologische Voruntersuchung. Übersichtsaufnahme in laterolateraler und ventrodorsaler Projektion eines gesunden Kaninchens. (@) Lena Carolin Voigt, Klinik für Heimtiere, Reptilien, Zier- und Wildvögel, Stiftung Tierärztliche Hochschule Hannover
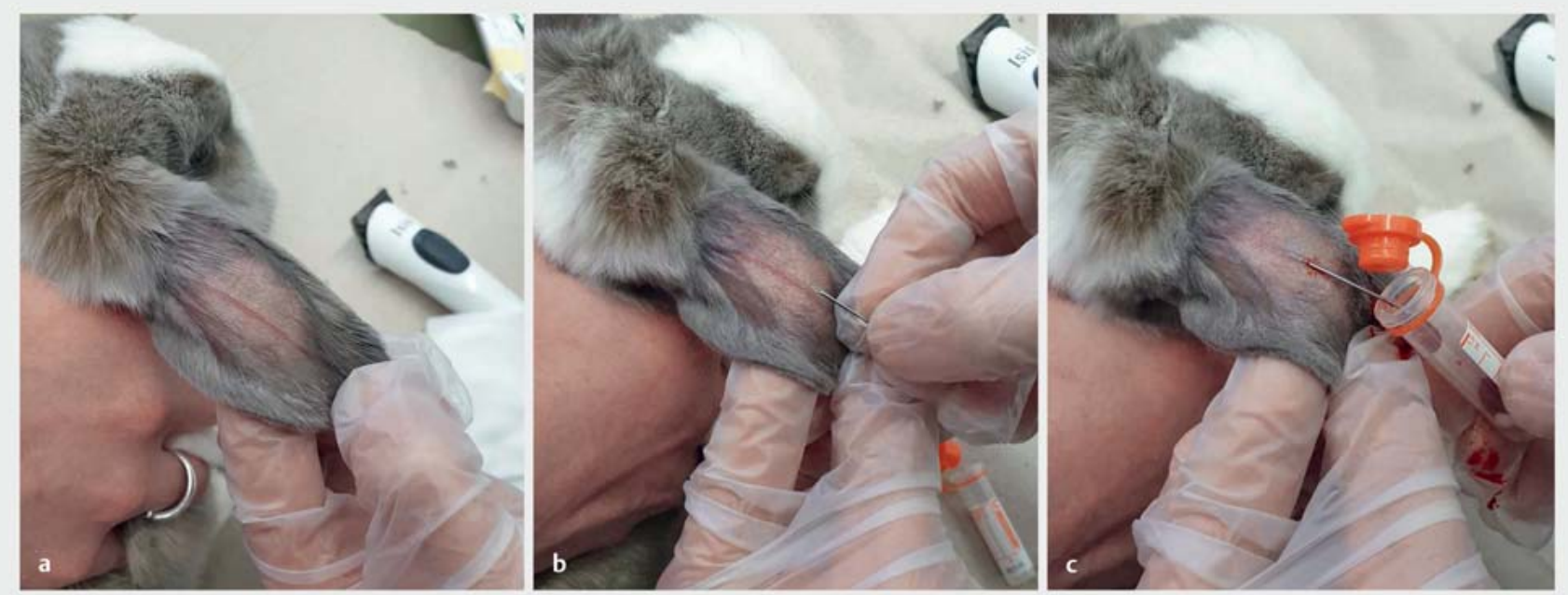

- Abb. 3 Blutentnahme bei einem Kaninchen an der A. auricularis als Teil der präanästhetischen Untersuchung. @ Lena Carolin Voigt, Klinik für Heimtiere, Reptilien, Zier- und Wildvögel, Stiftung Tierärztliche Hochschule Hannover 
Der Dehydratationszustand lässt sich mithilfe des Gesamteiweißes und des Hämatokrits leicht feststellen und kann so noch vor dem Eingriff behoben werden. Bei einer hochgradigen, akuten Anämie ist es sinnvoll, dem Patienten vor einem chirurgischen Eingriff Blut bereitzustellen bzw. noch vor dem Eingriff ein passendes Spendertier zu suchen. Sehr niedrige Gesamteiweißwerte führen zu einem geringen onkotischen Druck, sodass normale Ersatzlösungen als Infusion nicht ausreichend im Gefäßsystem gehalten werden können. In solchen Fällen sollte der Patient zusätzlich zu der Ersatzlösung einen kolloidalen Volumenersatz (Hydroxyethylstärke, Gelafundin 4\% Infusionslösung) erhalten. Ein niedriges Gesamteiweiß muss auch bei der Dosierung der Anästhetika berücksichtigt werden, da die Wirkstoffe bei niedrigem Plasmaeiweiß in größerer Menge ungebunden und damit wirksam vorliegen.

Ein Nieren- oder Leberpatient erhält ein nieren- oder leberschonenderes Protokoll als ein gesundes Heimtier. Zusätzlich ist diese Information für den Anästhesisten ausgesprochen wichtig. Patienten mit einer Lebererkrankung können einige Wirkstoffe nicht mehr gut verstoffwechseln. Diese sollten in der Medikation vermieden werden. Bei Nierenpatienten sollten nierenschädigende Medikamente ebenfalls vermieden werden, v.a. NSAID. In der Anästhesie muss zudem sehr genau auf den Blutdruck und eine adäquate Flüssigkeitstherapie geachtet werden. Die Nieren- und Leberfunktion ist aber nicht nur für eine komplikationslos verlaufende Allgemeinanästhesie von Bedeutung, sondern auch für eine schnelle und ruhige Aufwachphase, da diese Organe bei dem Abbau eine entscheidende Rolle spielen. Ist der Eingriff nicht lebensnotwendig und kann er ggf. verschoben werden, sollten die Patienten zunächst stabilisiert werden.

\section{ASA-Einstufung}

Die American Society of Anaesthesiologist (ASA) hat eine Klassifikation des präanästhetischen Allgemeinzustands ( $\vee$ Tab. 1) entwickelt, die sich mittlerweile auch in der Tiermedizin durchgesetzt hat [2]. Die Einteilung in die jeweilige ASA-Gruppe sollte für jeden Patienten vor jeder
Anästhesie erfolgen und dient der Risikoabschätzung und letztlich auch für die Besitzeraufklärung. Die Mortalität bei Kaninchen der ASA-Gruppe 3-5 liegt etwa 10mal höher $(7,4 \%)$ als bei Kaninchen aus der ASA-Stufe 1$2(0,7 \%)[1]$.

\section{Stabilisation und \\ Anästhesievorbereitung}

Es empfiehlt sich stets alle Kaninchen vor einer Anästhesie ihrem Allgemeinzustand entsprechend zu stabilisieren. Besonders Patienten, die nicht der Gruppe ASA 1-2 entsprechen, sollten vor jeder Allgemeinanästhesie zunächst hinsichtlich Kreislauf und Temperatur stabilisiert werden.

\section{Ruhige Umgebung}

Kaninchen sind sehr stressempfindliche Tiere. Der Transport, die fremde Umgebung, die Untersuchungen sowie Lärm und unbekannte Geräusche führen zu einem starken Anstieg der Katecholamin-Ausschüttung. Damit die Tiere jedoch möglichst ohne erhöhten Katecholaminspiegel in die Narkose gehen, empfiehlt es sich die Untersuchungen 1 Tag vorher durchzuführen und die Tiere an diesem Tag stationär aufzunehmen. So können sie sich in Ruhe akklimatisieren. Ist dies nicht möglich, sollten die Tiere zumindest einige Stunden an einem ruhigen Ort mit möglichst wenig Handling in der Klinik/Praxis zur Ruhe kommen können.

\section{Fütterung und Wärme}

Kaninchen dürfen vor der Narkose nicht gefastet werden, da ein Nahrungsentzug zu schweren Störungen des Säure-Basen-Haushalts und hochgradigen Hypoglykämien führen kann. Erbrechen ist bei Kaninchen anders als bei Hund und Katze nicht möglich. Schmerzen und Stress wirken sich negativ auf den sehr empfindlichen Magen-Darm-Trakt der Kaninchen aus und führen zu Inappetenz.

- Tab. 1 ASA-Stufen mit Erläuterungen zur richtigen Einteilung.

\begin{tabular}{|l|l|}
\hline ASA-Stufe & Allgemeinzustand \\
\hline ASA 1 & gesunder Patient - elektiver Eingriff, aus medizinischer Sicht nicht notwendig für das Wohlbefinden des Patienten (z. B. Kastration) \\
\hline ASA 2 & Patient mit geringgradig gestörtem Allgemeinbefinden, keine funktionelle Beeinträchtigung \\
\hline ASA 3 & $\begin{array}{l}\text { Patient mit schwerer Erkrankung, die die Aktivität einschränkt, aber nicht vollständig verhindert (z. B. extremes Alter, rassespezifisch, } \\
\text { symptomatische Herzerkrankung) }\end{array}$ \\
\hline ASA 4 & festliegender Patient mit lebensbedrohlicher Erkrankung (z. B. Sepsis, Organversagen) \\
\hline ASA 5 & $\begin{array}{l}\text { moribunder Patient, der erwartungsgemäß die nächsten 24 Stunden mit oder ohne Operation nicht überlebt (z. B. multiples Organ- } \\
\text { versagen) }\end{array}$ \\
\hline E & Notfallpatient (z. B. Magendrehung, Leberlappentorsion) \\
\hline
\end{tabular}




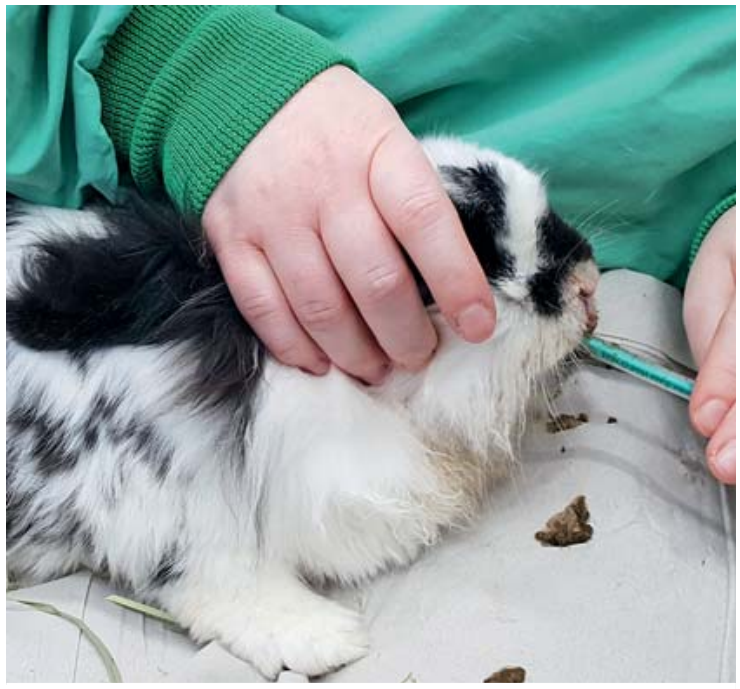

- Abb. 4 Handfütterung eines Kaninchens als Vorbereitung auf eine Anästhesie. ( $)$ Lena Carolin Voigt, Klinik für Heimtiere, Reptilien, Zier- und Wildvögel, Stiftung Tierärztliche Hochschule Hannover

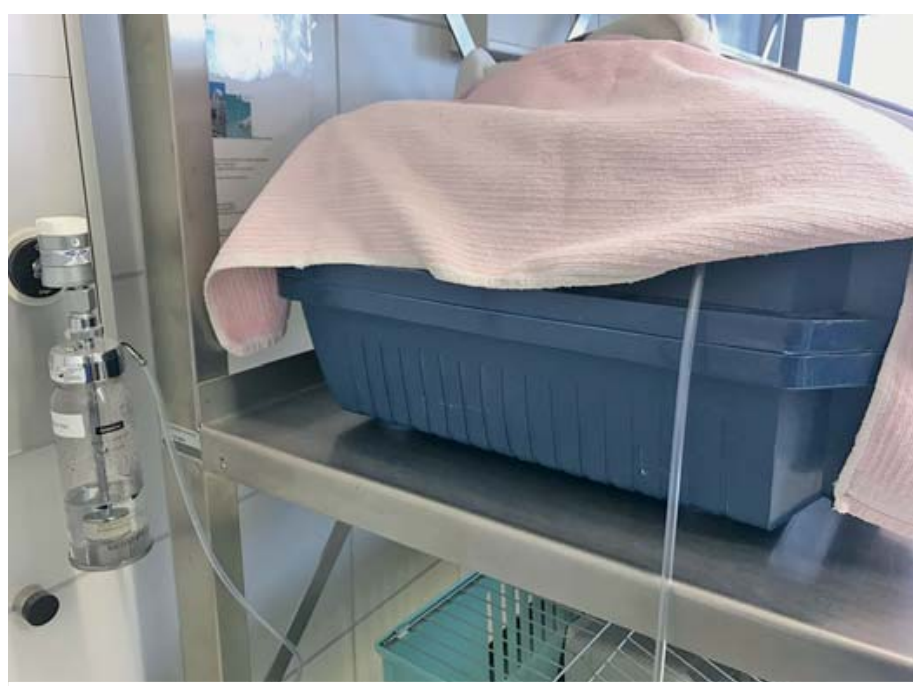

- Abb. 5 Stressfreie Präoxygenierung vor der Einleitung der Anästhesie in einer abgedeckten Transportbox. ( $)$ Lena Carolin Voigt, Klinik für Heimtiere, Reptilien, Zier- und Wildvögel, Stiftung Tierärztliche Hochschule Hannover
Merke

Inappetente Patienten müssen daher vor der Narkose unbedingt zugefüttert werden ( $\triangleright$ Abb. 4).

Um einen starken Wärmeverlust in der Anästhesie vorzubeugen, ist es stets empfehlenswert, die Kaninchen vor der Einleitung der Narkose vorzuwärmen (pre-warming). Dies ist z. B. mit einer Wärmematte möglich.

\section{Präoxygenierung}

Wie bei allen anderen Kleintieren empfiehlt es sich, die Kaninchen vor der Einleitung zu präoxygenieren. Das lindert eine unmittelbar nach der Gabe der Anästhetika entstehende Hypoxie bis die Tiere intubiert sind, da es meist unmittelbar nach der Einleitung zu einer respiratorischen Depression kommt. Dies geschieht vor der Einleitung der Narkose am stressärmsten in einer abgedeckten Transportkiste, in die Sauerstoff über einen Schlauch eingeleitet wird ( $\bullet$ Abb. 5). Nach der Einleitung der Narkose sollte Sauerstoff weiterhin über eine Maske bzw. während des Intubierens über einen vor die Nase gehaltenen Schlauch substituiert werden.

\section{Venenverweilkatheter}

Das Legen eines Venenverweilkatheters ( $\triangleright$ Abb. 6 ) ist, falls möglich, vor der Narkose durchzuführen. So kann bei einem Narkosezwischenfall schon während der Einleitung schneller reagiert werden. Beim Kaninchen eignet
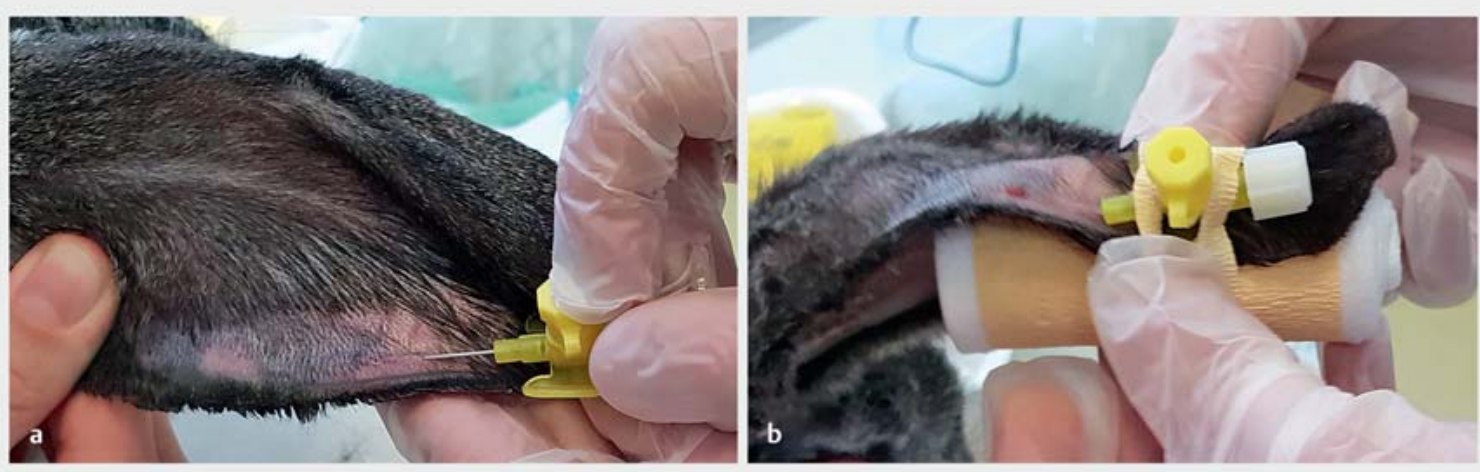

- Abb. 6 Legen eines Venenverweilkatheters vor der Anästhesieeinleitung in die V. auricularis lateralis. @ Lena Carolin Voigt, Klinik für Heimtiere, Reptilien, Zier- und Wildvögel, Stiftung Tierärztliche Hochschule Hannover 
sich die V. auricularis lateralis ( $\mathbf{A b b} \mathbf{6 a}$ ) für einen Venenverweilkatheter besonders gut. Alternativ können die V. cephalica antebrachii und die V. saphena ebenfalls verwendet werden.

\section{Fazit}

Eine gewissenhafte präanästhetische Untersuchung sowie eine gute, stressfreie Vorbereitung des Heimtiers auf die Narkose können das Anästhesierisiko deutlich senken. Erkrankungen, die für die bevorstehende Anästhesie von Bedeutung sind, können rechtzeitig identifiziert und zunächst behandelt werden. Des Weiteren beeinflussen die Ergebnisse der präanästhetischen Untersuchung ebenfalls die Wahl der Anästhetika und das Anästhesieverfahren. Um das gesamte Narkoserisiko besser abschätzen zu können, sollte jeder Patient mithilfe der präanästhetischen Untersuchung einer ASA-Gruppe zugeordnet werden.

\section{TIPP}

Die Klassifikation des präanästhetischen Allgemeinzustands der American Society of Anaesthesiologist (ASA) finden Sie auf der ASA-Webseite: www.asahq. org/standards-and-guidelines/asa-physical-statusclassification-system.
Korrespondenzadresse

\section{Dr. Lena Carolin Voigt}

Stiftung Tierärztliche Hochschule Hannover Klinik für Heimtiere, Reptilien, Zier- und Wildvögel

Bünteweg 9

30559 Hannover

Lena.Carolin.Voigt@tiho-hannover.de

\section{Literatur}

[1] Brodbelt DC et al. The risk of death: the confidential enquiry into perioperative small animal fatalities. Vet Anaesth Analg 2008; 35 (5): 365-373

[2] ASA. Online: www.asahq.org/standards-and-guidelines/asaphysical-status-classification-system. Letzter Zugriff: 05.03.2019

[3] Harcourt-Brown F. Anaesthesia and analgesia. In: Varga M, ed. Textbook of Rabbit Medicine. Oxford: Elsevier; 2002: 121-139

[4] Bonath K, Nolte I, Schniewind A et al. Food deprivation as preparation for anesthesia and aftercare-effect of fasting on the acid-base status and glucose concentration in the blood of rabbits of different body weight. Berl Munch Tierarztl Wochenschr 1982; 95 (7): 126-130

[5] Grint, N. Anaesthesia. In: Harcourt-Brown F, Chitty ], eds. BSAVA Manual of Rabbit Surgery, Dentistry and Imaging. Gloucester, Quedgeley, UK: Blackwell; 2013: 1-25

\section{Bibliografie}

DOI https://doi.org/10.1055/a-0851-9178

Kleintier konkret 2019; 22: 9-14

(c) Georg Thieme Verlag KG Stuttgart · New York ISSN 1434-9132 